July 5, 2021

HU-TFT-94-26

\title{
Dirac neutrinos and primordial magnetic fields
}

\author{
Kari Enqvist $\prod^{\Upsilon}$ \\ Research Institute for Theoretical Physics, University of Helsinki, P.O. Box 9, \\ SF-00014 Helsinki, Finland \\ and

\section{A.I. Rez[ and V.B. Semikoz} \\ The Institute of the Terrestrial Magnetism, the Ionosphere and Radio Wave \\ Propagation of the Russian Academy of Sciences, IZMIRAN,Troitsk, Moscow \\ region, 142092, Russia.
}

\begin{abstract}
We consider random primordial magnetic fields and discuss their dissipation, coherence length $L_{0}$, scaling behaviour and constraints implied by the primoridal nucleosynthesis. Such magnetic fields could excite the right-helicity states of Dirac neutrinos, with adverse consequences for nucleosynthesis. We present solutions to the spin kinetic equation of a Dirac neutrino traversing a random magnetic field in the cases of large and small $L_{0}$, taking also into account elastic collisions. Depending on the scaling behaviour and on the magnetic coherence length, the lower limit on the neutrino magnetic moment thus obtained could be as severe as $10^{-20} \mu_{B}$.
\end{abstract}

\footnotetext{
${ }^{1}$ enqvist@phcu.helsinki.fi; on a leave of absence from NORDITA, Blegdamsvej 17, DK-2100 Copenhagen, Denmark

${ }^{2}$ rez@charley.izmiran.rssi.ru; rez@izmiran.troitsk.su

3semikoz@charley.izmiran.rssi.ru; semikoz@izmiran.troitsk.su
} 


\section{Introduction}

The phase transitions of the very early universe may have generated primordial magnetic fields, which could play an important role in cosmology. Large primordial fields can survive to this day and provide a seed field for the galactic dynamo mechanism [1] which amplifies the seed to produce the observed galactic magnetic fields. Several suggestions have been made as to the possible mechanisms which could produce large primordial fields [2]. For instance, large fluctuations at the electroweak phase transition might be responsible [3], and it has been argued [4] that after a proper statistical averaging one could actually obtain seed fields of the required magnitude [5], about $10^{-18}$ G. A more exotic possibility which also seems to work is based on the observation that in Yang-Mills theories the vacuum may have a permanent magnetic field, which is imprinted on the comoving plasma already at the GUT scale [6].

Primordial nucleosynthesis is sensitive to magnetic fields, which modify both the Hubble rate and the rates of the reactions that help to build up the light elements [7]. Primordial nucleosynthesis may also be affected in another way [8] provided neutrinos are Dirac particles. In that case the right-helicity component of the neutrino can be excited and brought into thermal equilibrium by scattering of the left-helicity neutrinos off the magnetic field, thus changing the effective number of degrees of freedom.

In a recent estimate of the primordial helium abundance [9] the allowed number of the additional neutrino species was reduced to $\Delta N_{\nu}=N_{\nu}-3 \simeq 0.1$. There is some uncertainty in this estimate due to the unkown magnitude of systematic errors in the observed abundances, but even allowing for very conservative systematic errors, $\Delta N_{\nu}$ is definitely less than 1 . Thus the coupling of a Dirac neutrino to a primordial magnetic field should be weak enough not to equilibrate the right-helicity states below the QCD phase transition temperatures. This gives rise to a bound on the combination $\mu_{\nu} B$, where $\mu_{\nu}$ is the magnetic moment of the neutrino.

The right-handed neutrino production rate is proportional to the neutrino helicity flip probability, which may be calculated by considering neutrino spin rotation in a medium with an external magnetic field. The complicated time evolution of the neutrino spin can be described in terms of a relativistic kinetic equation (RKE), which has been derived in [10] and was extended to account for the elastic collisions in [11]. The helicity rotation of a light Dirac neutrino traversing a magnetic field is determined by forward scattering off the field. Simultaneously, the neutrino interacts with all the particles in the plasma via reactions that for light neutrinos can be taken to conserve helicity. An essential feature in deriving the RKE is averaging over the (random) magnetic field, the procedure of which we improve in the present paper in order to 
discuss also small scale fields. In what follows, we shall limit ourselves to the Standard Model but assume that the neutrinos have Dirac masses.

If we neglect the magnetic moment of the Standard Model Dirac neutrino, in the ultrarelativistic limit the dispersion relation, in hot plasma and in the presence of a magnetic field, reads $E(q) \approx q+V$. Here $V$ is the neutrino interaction potential which is determined by the neutrino forward scattering amplitude and which consists of two parts [12]:

$$
V=V^{(v e c)}+V^{(a x i a l)}
$$

$V^{(\text {axial })}$ includes contributions which are due to the fact that in the presence of a magnetic field the charged background is actually magnetized. This turns out to be an important effect. The interaction potential $V^{(v e c)}$ is generated by the mean vector current $\sim\left\langle\bar{\psi}_{a} \gamma_{\mu} \psi_{a}\right\rangle_{0}$, and at the temperature $m_{e} \ll T \ll T_{\mathrm{QCD}}$ it reads [13]

$$
V^{(v e c)} \approx 3.4 \times 10^{-20}\left(\frac{T}{\mathrm{MeV}}\right)^{5} \mathrm{MeV}
$$

$V^{(a x i a l)}$ is generated by the mean axial current of the magnetized leptons $\sim\left\langle\bar{\psi}_{l} \gamma_{\mu} \gamma_{5} \psi_{l}\right\rangle_{0}$ and is given by 12

$$
V^{(a x i a l)}=\mu_{e f f} \frac{\mathbf{q} \cdot \mathbf{B}}{q}+\frac{\mu_{e f f}^{2}}{2 q}\left(B^{2}-\frac{(\mathbf{q} \cdot \mathbf{B})^{2}}{q^{2}}\right),
$$

where the quantity $\mu_{\text {eff }}$ is defined by

$$
\mu_{e f f}=\frac{e G_{F}\left(-2 c_{A}\right) T \ln 2}{\sqrt{2} \pi^{2}} \approx 6 \times 10^{-13}\left(-2 c_{A}\right) \mu_{B}\left(\frac{T}{\mathrm{MeV}}\right),
$$

and $c_{A}=\mp 0.5$ is the axial constant in the weak lepton current (upper sign for $\nu_{\mathrm{e}}$, lower for $\left.\nu_{\mu, \tau}\right)$. Here $\mu_{B}=e / 2 m_{e}$ is the Bohr magneton. In what follows we may safely neglect the last term in Eq. (3).

The size of the random magnetic field domain $L_{0}$ influences crucially the neutrino spin behaviour. For large-scale random magnetic field with $L_{0} \gg V^{-1}$ the dominant mode of evolution is spin oscillation. For small scale magnetic fields (which is perhaps a more realistic alternative in the early universe) there also appears aperiodic spin motion which effectively converts $\nu_{L}$ to $\nu_{R}$. We discuss $L_{0}$, dissipation, conductivity and general constraints on primordial magnetic fields in section 2 . In section 3 we derive a cosmological limit on the neutrino magnetic moment in the case of a large scale magnetic field, taking into account elastic collisions and improving on previous treatments. Section 4 introduces an averaging procedure which is suitable for small

\footnotetext{
${ }^{4}$ Note that this effective magnetic moment has no relation with the anomalous neutrino magnetic moment.
} 
scale magnetic fields, and we provide a limit on the neutrino magnetic moment also in this case. Section 5 contains our comments and a discussion of the meaning of the results.

\section{Direct constraints on magnetic fields}

The early universe is an excellent conductor, and a primordial magnetic field, if such existed at any time, is imprinted on the comoving plasma which will retain the field. The magnetic flux is conserved so that the magnetic field scales with the expansion of the universe as $B \sim R^{-2}$. The strength of a random magnetic field depends also on how it scales over physical distances. If the field were to perform random walk in 3 -d volume, the scaling would be $B \sim N^{-3 / 2}$, where $N$ is the number of steps. An argument based on the statistical independence of conserved flux elements gives rise to a $B \sim N^{-1}$ scaling. Whether the magnetic flux actually is completely uncorrelated in the neighbouring unit cells is an unsolved issue. In [4] it was argued that if the magnetic field domains are uncorrelated, a proper statistical averaging produces a field with $\langle B\rangle=0$ and a root-mean-square field $B_{r m s} \equiv \sqrt{B^{2}} \sim N^{-1 / 2}$. In the present paper we take a more phenomenological view and assume merely that there exists a random magnetic field with the scaling

$$
B(L, t)=B_{0}\left(\frac{R_{0}}{R(t)}\right)^{2}\left(\frac{L_{0}}{L}\right)^{p},
$$

where $L$ and $L_{0}$ are two comoving physical scales, and $p$ is essentially an unknown parameter; we shall focus mainly on the choices $p=1 / 2,1,3 / 2$. We shall view $B(L, t)$ in Eq. (5) as a root-mean-squared field with $\left\langle B_{j}\right\rangle=0$. We shall also assume isotropy so that $\left\langle B_{i} B_{j}\right\rangle=0$ for $i \neq j$. If we further assume that the magnetic domains are uncorrelated we may write

$$
\left\langle B_{i}(\mathbf{x}) B_{j}(\mathbf{y})\right\rangle=(2 \lambda)^{-1} \delta_{i j} \delta^{(3)}(\mathbf{x}-\mathbf{y})
$$

where the length $\lambda$ is determined by the domain size $L_{0}$ and the rms field (5) at the horizon scale $L=l_{H}(T)$ :

$$
\begin{aligned}
\lambda^{-1} & =\frac{3}{\pi(3-2 p)} B_{r m s}^{2}\left(L=l_{H}\right) L_{0}^{3}, \quad p \neq 3 / 2 \\
\lambda^{-1} & =\frac{3}{\pi}\left(\ln \frac{l_{H}}{L_{0}}\right) B_{r m s}^{2}\left(L=l_{H}\right) L_{0}^{3}, \quad p=3 / 2 .
\end{aligned}
$$


We may prove Eq. (7) by recalling the general fluctuation theory formula甲 [14]

$$
\left\langle B_{i}(\mathbf{k}) B_{j}\left(\mathbf{k}^{\prime}\right)\right\rangle=(2 \pi)^{3}\left\langle\mathbf{B}^{2}\right\rangle_{\mathbf{k}} \delta^{(3)}\left(\mathbf{k}+\mathbf{k}^{\prime}\right)\left(\delta_{i j}-\hat{k}_{i} \hat{k}_{j}\right) .
$$

If we substitute the Fourier transform $B_{i}(\mathbf{x})=\int d^{3} k \exp (i \mathbf{k x}) B_{i}(\mathbf{k}) /(2 \pi)^{3}$ into the Anzatz (6) and use the rule Eq. (8), we obtain a constant spectrum

$$
\left\langle\mathbf{B}^{2}\right\rangle_{\mathbf{k}}=\frac{3}{2} \frac{1}{2 \lambda}=\text { const }
$$

where the factor $3 / 2$ arises because of a different delta-function implementation in Eq. (8) as compared with Eq. (6) .

Now we can express the parameter $\lambda$ in Eq. (6) via the field in Eq. (5). Let us first note that the mean magnetic field energy within a horizon volume,

$$
\rho_{B}=\frac{1}{V_{H}} \int d^{3} r \frac{B_{r m s}^{2}(r)}{2}=\frac{1}{2} \int \frac{d^{3} k}{(2 \pi)^{3}}\left\langle\mathbf{B}^{2}\right\rangle_{\mathbf{k}}
$$

depends 9 on a cut-off wave number $k_{\max }$ :

$$
\frac{2 \pi}{V_{H}} B_{0}^{2}\left(\frac{T}{T_{0}}\right)^{4} \int_{L_{0}}^{l_{H}} r^{2} d r\left(\frac{L_{0}}{r}\right)^{2 p}=\frac{k_{\max }^{3}}{16 \lambda \pi^{2}} .
$$

Physically $k_{\max }=2 \pi / L_{0}$ corresponds to a minimum size of the inhomogeneity, and is given as in 10 by

$$
\frac{3}{5} k_{\max }^{2}=\frac{\int k^{2} d^{3} k\left\langle\mathbf{B}^{2}\right\rangle_{\mathbf{k}} /(2 \pi)^{3}}{\left\langle\mathbf{B}^{2}\right\rangle_{\mathbf{x}=0}} .
$$

Here the factor $3 / 5$ arises by using Eqs. (9) and (10).

Substituting Eq. (12) into Eq. (11) we arrive at the relations Eq. (7) between $\lambda$, the domain size $L_{0}$ and the field $B_{r m s}$ at the horizon scale $l_{H}$ :

$$
\lambda^{-1}=\frac{3}{\pi(3-2 p)} B_{0}^{2}\left(\frac{T}{T_{0}}\right)^{4}\left(\frac{L_{0}}{l_{H}}\right)^{2 p} L_{0}^{3}=\frac{3}{\pi(3-2 p)} B_{r m s}^{2}\left(L=l_{H}\right) L_{0}^{3}, \quad p \neq 3 / 2,
$$

and similarly for $p=3 / 2$.

Let us now turn to the constraints on the domain size $L_{0}$ and on the magnetic field strength in the plasma of the early universe. We begin by discussing the dissipation of primordial magnetic fields.

\footnotetext{
${ }^{5}$ For real fields we use the + sign in the $\delta$-function argument instead of the usual - sign for the conjugated magnetic field components.

${ }^{6}$ The second equality here has the sense of the mean field energy density $\frac{1}{2}\left\langle\mathbf{B}^{2}\right\rangle_{\mathbf{x}=0}=$ $\frac{1}{2} \int \frac{d^{3} k}{(2 \pi)^{3}}\left\langle\mathbf{B}^{2}\right\rangle_{\mathbf{k}}$ for fluctuating fields [15. The first equality in Eq. (10) has this meaning as a definition. Of course, for any index $p$ in Eq. (5) the magnetic field does not affect the expansion of the universe because $\rho_{B} \ll \rho_{\gamma} \sim T^{4}$. We use here the Gauss units in the which for the uniform field $\left\langle\mathbf{B}^{2}\right\rangle_{\mathbf{k}}=B_{0}^{2}(2 \pi)^{3} \delta^{(3)}(\mathbf{k})$ the magnetic field energy density is given by $\rho_{B}=B_{0}^{2} / 2$.
} 
Conductivity in the early universe, although large, is not infinite, and accordingly there will be some ohmic dissipation, starting from small length scales, $L_{0} \leq L_{\text {diss }}$. The dissipation scale is given by

$$
L_{\text {diss }}=\sqrt{\frac{t}{4 \pi \sigma_{c}}},
$$

where $t$ is the age of the universe and $\sigma_{c}$ is conductivity, which for isotropic relativistic electron gas reads

$$
\sigma_{c}=\frac{\omega_{p}^{2}}{4 \pi \sigma n_{e}}=\frac{T}{\pi \alpha L_{c}}
$$

Here $\omega_{p}=\sqrt{4 \pi \alpha n_{e} / T}$ is the plasma frequency and $\sigma \approx \pi \alpha^{2} L_{c} / T^{2}$ is the collision cross section and $L_{c} \sim 3$ is the Coulomb logarithm. We then find that the dissipation length below $T_{\mathrm{QCD}}$ is given by

$$
L_{\text {diss }}^{r e l}=0.1 g_{*}^{-1 / 4}\left(\frac{\mathrm{MeV}}{T}\right)^{3 / 2} \mathrm{~cm}
$$

The finite isotropic conductivity (15) reflects the properties of the plasma at large scales when we may neglect the random magnetic field influence upon relativistic plasma. We may thus use the estimate $(\sqrt{16})$ to obtain a conservative lower bound on the domain size $L_{0}$.

It is worth noting that within a domain volume with a uniform magnetic field where plasma is highly anisotropic, the dissipation length is greatly reduced with respect to Eq. (17) because of substantial increase of conductivity, i.e. a strong magnetic field tends to sustain itself within a given domain. Indeed, for relativistic electron-positron plasma in a strong uniform magnetic field, which is much larger than the quantizing magnetic field $B_{c}=m_{e}^{2} / e=4.41 \times 10^{13} \mathrm{G}$, conductivity is given by

$$
\sigma_{c}=\frac{\omega_{p}^{2}}{4 \pi \sigma n_{e}}=\frac{T\left(B / B_{c}\right)}{4 \pi \alpha C_{E}^{2}} .
$$

Here $\sigma \approx 4 C_{E}^{2} \pi \alpha^{2}\left(B_{c} / B\right) / T^{2}$ is the $e^{+} e^{-}$collision cross section [16] for the electrons and the positrons that occupy the lowest Landau level, and $C_{E} \approx 0.577$ is the Euler constant. The dissipation length within a domain with a strong uniform field is given by

$$
L_{\text {diss }}^{\text {anis }}=\frac{3.5 \times 10^{-3} \mathrm{~cm}}{g_{*}^{1 / 4}}\left(\frac{\mathrm{MeV}}{T}\right)^{3 / 2} \times\left(\frac{B_{c}}{B}\right)^{1 / 2} .
$$

Let us note that within one domain with a uniform magnetic field the ratio $B / B_{c}$ can be interchanged with the temperature ratio, $B / B_{c} \sim(T / \mathrm{MeV})^{2}$. The dependence of the magnetic field on the temperature reflects the magnetic flux conservation. Here we 
neglected the small contribution to the plasma conductivity produced by the collisionless one-photon processes in a strong external magnetic field $\left(\gamma \leftrightarrow e^{+} e^{-}, \quad e^{ \pm} \leftrightarrow e^{ \pm} \gamma\right)$. This conductivity is calculated from the imaginary part of the polarization tensor in magnetic field [17] and can be estimated as

$$
\sigma_{c}^{\text {one-photon }} \simeq \frac{\alpha\left(B / B_{c}\right) m_{e}^{2}}{4 \pi T} \ll \sigma_{c}
$$

where $\sigma_{c}$ is given by Eq. (17).

Comparing the relativistic dissipation length (16) with the analogous result for anisotropic plasma (18) we find that one can neglect the magnetic field dissipation at small scales since domains with sizes $L_{0}(T) \geq L_{\text {diss }}^{r e l}(T) \gg L_{\text {diss }}^{\text {anis }}$ survive.

In the non-relativistic case, which is relevant for the recombination time $t \simeq 10^{12} \mathrm{sec}$ $\left(T_{\text {rec }} \sim 0.4 \mathrm{eV}\right)$ and for the BBN time $t \sim 1 \min \left(T_{B B N} \sim 0.1 \mathrm{MeV}\right)$, one obtains a different expression for the dissipation length,

$$
L_{\text {diss }}^{\text {non-rel }}=0.1 g_{*}^{-1 / 4}\left(\frac{\mathrm{MeV}}{T}\right)^{7 / 4} \mathrm{~cm}
$$

where we have used the conductivity of non-relativistic isotropic hydrogen plasma 18

$$
\sigma_{c}=\frac{4 \sqrt{2} T^{3 / 2}}{\pi^{3 / 2} \alpha L_{c} m_{e}^{1 / 2}} .
$$

The difference between this and the relativistic expression (15) follows from: (i) the Debye screening for the forward ee or $e p$ scattering in isotropic non-relativistic plasma with the Coulomb logarithm $L_{c} \sim 10$, and (ii) the fact that in the non-relativistic case the velocity of the electrons is the thermal velocity $v_{T_{e}}=\sqrt{T / m_{e}} \neq 1$.

The dissipation length $(20)$ at the recombination time is about $\sim 10^{10} \mathrm{~cm}$ which translates to the BBN time $t \sim 1 \min \left(T_{B B N} \sim 0.1 \mathrm{MeV}\right)$ as $L_{0}^{\min }\left(T_{B B N}\right)=10^{10} \mathrm{~cm} \times$ $\left(T_{\text {rec }} / T_{B B N}\right) \simeq 4 \times 10^{4} \mathrm{~cm}$. The domains which at that time are larger than this scale, $L_{0} \gtrsim L_{0}^{\min }\left(T_{B B N}\right)$, survive after the recombination time, and such a relic field could be a seed field for the dynamo enhancement of the galactic magnetic field. This requirement can be rewritten as (see also [7])

$$
L_{0} \geq L_{0}^{\min }=10^{3} \mathrm{~cm} \times\left(\frac{\mathrm{MeV}}{T}\right) .
$$

Let us note that the local dissipation length calculated at the same BBN time from Eq. (20) is significantly smaller, $L_{\text {diss }}^{\text {non-rel }}\left(T_{B B N}\right) \sim 5 \mathrm{~cm}$, i.e. domains with sizes within the region $5 \mathrm{~cm} \lesssim L_{0}\left(T_{B B N}\right) \lesssim L_{0}^{\min }\left(T_{B B N}\right) \sim 10^{4} \mathrm{~cm}$ continue to expand after the BBN time but they dissipate before the recombination time and do not contribute to the relic seeding of galactic magnetic field. 
For instance, the relativistic dissipation length (16) at the temperature corresponding to the electron neutrino decoupling with matter, $T \sim T_{d} \sim 2 \mathrm{MeV}$, is even smaller,

$$
L_{\text {diss }}^{r e l}(T \sim 2 \mathrm{MeV}) \sim 10^{-2} \mathrm{~cm}
$$

or $\sim 0.3 \mathrm{~cm}$ after its expansion till the BBN time. Therefore, all the domains that have survived in the hot plasma but have at $T_{B B N}$ a size within the range $0.3 \mathrm{~cm} \lesssim L_{0}\left(T_{B B N}\right) \lesssim 5 \mathrm{~cm}$, in fact dissipate before $T_{B B N}$. On the other hand, all the domains that survive after the nucleosynthesis but have a size less than $10^{4} \mathrm{~cm}$ at $T_{B B N}$ dissipate before the recombination time.

Accounting for the expansion of the domains, we find that if

$$
L_{0}(T) \gtrsim L_{0}^{m i n^{\prime}}=10^{-2} \mathrm{~cm} \times\left(\frac{\mathrm{MeV}}{T}\right),
$$

such domains are guaranteed to survive at any relativistic temperature; however, they dissipate before the BBN time if their scale $L_{0}\left(T_{B B N}\right)$ was less than $L_{d i s s}^{\text {non-rel }}\left(T_{B B N}\right)=$ $5 \mathrm{~cm}$.

Primordial nucleosynthesis considerations can further be used to constrain the strength of the magnetic field. If large enough, a magnetic field would affect the expansion rate of the universe as well as the rates of the various reactions that are involved in building up the abundances of light elements. Including these effects in a nucleosynthesis code Cheng et al. find in a recent study [7] an upper bound

$$
B_{\mathrm{NS}} \equiv B\left(10^{4} \mathrm{~cm}, 1 \mathrm{~min}\right)<10^{11} \mathrm{G}
$$

This is a very useful constraint, as will become evident shortly.

The energy density in the magnetic field within the causal horizon is given by

$$
\rho_{B}=\frac{2 \pi B_{0}^{2}}{V_{H}}\left(\frac{R}{R_{0}}\right)^{4} \int_{L_{0}}^{l_{H}} d r r^{2}\left(\frac{L_{0}}{r}\right)^{2 p},
$$

where $V_{H}$ is the causal horizon volume. (At $T_{\mathrm{EW}} \simeq 100 \mathrm{GeV}$ the causal horizon size is $l_{H} \simeq 1.4 \mathrm{~cm}$ ). A reasonable requirement is that the energy of the magnetic field fluctuations should not exceed the free electromagnetic energy, so that $\rho_{B} \lesssim T^{4}$. This is not in disagreement with the nucleosynthesis constraint Eq. (25) at any scale below $T_{\mathrm{EW}}$. If we assume, as is natural, that the random field fluctuations are bounded by $B\left(L_{0}, t\right) \lesssim \mathcal{O}\left(T^{2}\right)$, then $\rho_{B} \lesssim T^{4}$ for $l_{0}<l_{H}$ for all $p$ under consideration.

If we assume that the observed galactic magnetic fields are due to a large scale dynamo effect [1], seeded by the primordial field, then we may set a lower bound on the strength of the cosmological seed field $B_{\text {seed }}$ (this is further enhanced by a factor of $10^{4}$ by the collapse of the protogalaxy) [19]. Numerical simulations of the field growth 
appear to imply that the seed field must be sufficiently large for the dynamo to work [0], and one should require that at $t \simeq 10^{10} \mathrm{yr}$ and at the intergalactic scale $L_{\text {Gal }}=100$ $\mathrm{kpc}, B_{\text {seed }} \equiv B\left(L_{\mathrm{Gal}}, 10^{10} \mathrm{yr}\right) \gtrsim 10^{-18} \mathrm{G}$. This bound can be made even tighter provided we assume that the observed field reversal between the Orion and Sagittarius arms [20] is related to the dynamo. To produce a field reversal, the seed field should have been relatively strong [21], $B_{\text {seed }} \gtrsim 10^{-11} \mathrm{G}$. Such reversal has only been observed in the Milky Way and may thus not be a generic feature.

From Eq. (25) it follows that

$$
B_{\text {seed }}=B_{\mathrm{NS}}\left(\frac{1 \mathrm{~min}}{t_{*}}\right)\left(\frac{t_{*}}{t_{\text {now }}}\right)^{4 / 3}\left(\frac{10^{4} \mathrm{~cm}}{L_{\mathrm{Gal}}}\right)^{p}<1.8 \times 2.4^{p} \times 10^{-7-11 p} \mathrm{G},
$$

where we have assumed that the change to matter dominated universe takes place at $t_{*} \simeq 8750 \mathrm{yr}$, corresponding to $\Omega h^{2} \simeq 0.4$. Therefore, the case $p=3 / 2$, which translates into the bound $B_{\text {seed }}<2 \times 10^{-23} \mathrm{G}$, cannot provide a seed field large enough for the galactic dynamo, no matter what mechanism generated the field in the first place. If $p=1$ we obtain $B_{\text {seed }}<4 \times 10^{-18} \mathrm{G}$ but given the large theoretical uncertainties, this case could still be compatible with the galactic dynamo. Finally, if the observed field reversals in the Milky Way are really related to the dynamo, then only the $p=1 / 2$ case remains marginally compatible.

It has been argued [22] that in magnetic fields of the order of $B \approx 10^{18} \mathrm{G}$ the neutron becomes stable against $\beta$-decay and that for somewhat larger fields proton becomes unstable to a decay into a neutron. If $B \lesssim T^{2}$ at all scales, this effect would not be important for nucleosynthesis. It is also irrelevant for the problem of excitation of the wrong-helicity neutrino states because neutrino spin flip is determined by the field strength at $T_{\mathrm{QCD}}$ and at scales of the order of the weak collision length $L_{W}$. Indeed, we may write the the nucleosynthesis bound (25) as

$$
B\left(L_{W}, T_{\mathrm{QCD}}\right) \lesssim 8 \times 10^{17} \times 13^{-p} \mathrm{G} \lesssim 2 \times 10^{17} \mathrm{G}
$$

Here we have used the estimate $L_{W} \approx 1.6 \times 10^{-4} \mathrm{~m}$. Considering the inherent uncertainties, we may then well adopt the latter figure in Eq. (28) as a conservative bound at $T_{\mathrm{QCD}}$ for all $p$.

Finally, note that the evolution of a magnetic field in an expanding Universe is determined by MHD which should explicitly yield the domain size $L_{0}$ as well as the topological index $p$ in Eq. (5), both of which are crucial for the neutrino propagation in a medium with a random field. We shall use the phenomenological model (5) for case of uncorrelated 3-d fields in Eq. (6) with an arbitrary index $p$. 


\section{$3 \quad$ Neutrino propagation in medium with large-scale random magnetic field}

Neutrino spin-flip in a magnetic field can affect the Big Bang nucleosynthesis of light elements because of the appearance of an additional gravitating relativistic component in the plasma. The nucleosynthesis limit on the extra degrees of freedom at the time of nucleosynthesis is [9], in units of relativistic two-component neutrinos, $\Delta N_{\nu} \lesssim 0.1$ (see also [23]). Indeed, the wrong-helicity neutrinos will be abundant at the time when the neutron-to-proton ratio freezes at $T \simeq 0.7 \mathrm{MeV}$, violating the nucleosynthesis bound, unless they decouple before the QCD phase transition. Then their relative number densities will be diluted to acceptable levels by the heating of the particles still in equilibrium.

In the early universe the production rate of the wrong-helicity neutrinos is given by $\Gamma_{L \rightarrow R}=\left\langle P_{\nu_{L} \rightarrow \nu_{R}}\right\rangle \Gamma_{W}^{\text {tot }}$, where $\Gamma_{W}^{\text {tot }}$ is the total weak collision rate, and in the absence of collisions the averaged conversion probability reads

$$
\left\langle P_{\nu_{L} \rightarrow \nu_{R}}\right\rangle=\frac{1}{2} \frac{\tilde{H}_{\perp}^{2}}{\omega^{2}}
$$

where $\tilde{H}_{\perp}=2 \mu_{\nu} B_{\perp}$ is the field perpendicular to the neutrino propagation, $\mu_{\nu}$ is the neutrino magnetic moment and

$$
\omega^{2}=2 \tilde{H}_{\perp}^{2}+V^{2}+6 L_{0}^{-2} / 5
$$

is the spin rotation frequency. Note that it depends both on the value and scale of the random field. In a uniform constant field one would find $\omega^{2}=\tilde{H}_{\perp}^{2}+V^{2}$ in the expression (29). In a realistic case $V \gg \tilde{H}_{\perp}$.

To find out $\Gamma_{L \rightarrow R}$, let us consider the electron neutrino for definiteness. Then at $T=T_{\mathrm{QCD}}$ one should include the following processes: $\nu_{\mathrm{e}} \bar{\nu}_{\mathrm{e}} \rightarrow l^{+} l^{-}, \nu_{\alpha} \bar{\nu}_{\alpha}, q \bar{q} ; \nu_{\mathrm{e}} l^{ \pm} \rightarrow$ $\nu_{\mathrm{e}} l^{ \pm} ; \nu_{\mathrm{e}} \nu_{\alpha} \rightarrow \nu_{\mathrm{e}} \nu_{\alpha} ; \nu_{\mathrm{e}} \bar{\nu}_{\alpha} \rightarrow \nu_{\mathrm{e}} \bar{\nu}_{\alpha} ; \nu_{\mathrm{e}} q \rightarrow \nu_{\mathrm{e}} q ; \nu_{\mathrm{e}} \bar{q} \rightarrow \nu_{\mathrm{e}} \bar{q} ; \nu_{\mathrm{e}} e^{+} \rightarrow u \bar{d} ; \nu_{\mathrm{e}} \mu^{-} \rightarrow$ $\nu_{\mu} \mu^{+} ; \nu_{\mathrm{e}} \bar{\nu}_{\mu} \rightarrow \mu^{-} e^{+}$. Here the notation is: $\alpha=\mathrm{e}, \mu, \tau ; l=\mathrm{e}, \mu ; q=u$, d. Adding up all these processes we find the thermally averaged electron neutrino collision rates to be

$$
\Gamma_{W}^{\mathrm{tot}}\left(T_{\mathrm{QCD}}\right) \approx 30 G_{F}^{2} T_{\mathrm{QCD}}^{5} ; \quad \Gamma_{W}^{\mathrm{el}}\left(T_{\mathrm{QCD}}\right) \approx 2 G_{F}^{2} T_{\mathrm{QCD}}^{2} .
$$

Here we have neglected the decays and inverse decays, whose contributions are small. The main contribution to Eq. (31) comes from the charged current processes involving quarks. For $\nu_{\mu}$ and $\nu_{\tau}$ the rate is slightly different.

Requiring $\Gamma_{L \rightarrow R} \lesssim H$ at $T=T_{\mathrm{QCD}}$ where $H=\sqrt{8 \pi^{3} g_{*}(T) / 90} T^{2} / M_{P l}$ is the Hubble parameter, one obtains a constraint on the product of the Dirac neutrino magnetic 
moment and the mean squared random field $B=\left\langle B^{2}\right\rangle^{1 / 2}$ [8]. Here we adopt the value $g_{*}\left(T_{\mathrm{QCD}}\right) \simeq 63$, which includes also the effects due to the non-relativistic species [24]. The limit thus obtained does not depend on any model for the random field, and one finds that

$$
\mu_{\nu} B\left(T_{\mathrm{QCD}}, L_{W}\right) \lesssim 4 \times 10^{2} \mu_{B}\left(\frac{T_{\mathrm{QCD}}}{200 \mathrm{MeV}}\right)^{7 / 2} \mathrm{G} .
$$

The result (32) is true in collisionless regime if the random domain size $L_{0}$ is larger than the neutrino spin oscillation length $l_{\text {osc }} \simeq V^{-1}$ with

$$
l_{\text {osc }}=10^{-2} l_{H}(T / \mathrm{MeV})^{-3},
$$

where $l_{H} \sim M_{P l} / T^{2}$ is the horizon length. For small domains, $L_{0} \ll l_{\text {osc }}$, neutrino spin cannot follow the random direction of the magnetic field and the neutrino spin rotation effectively ceases [10]. The limit (32) is slightly different from the one presented in [19] because of the inclusion of the quarks in the total collision rate.

If we take into account elastic neutrino collisions with charged particles in the background plasma, the analogous consideration starts from the RKE for the $z$-component of the neutrino spin $S_{z}(t)=2 P_{\nu_{L} \leftrightarrow \nu_{R}}(t)-1$. This is an integro-differential equation and given by [1]

$$
\begin{gathered}
\frac{d S_{z}(t)}{d t}=-2 \int_{0}^{t} \exp \left[-\int_{t_{1}}^{t} \nu_{\perp}\left(t_{2}\right) d t_{2}\right] \times\left[\tilde{H}_{-}(t) \tilde{H}_{+}\left(t_{1}\right) \exp \left(i \int_{t_{1}}^{t} V\left(t_{2}\right) d t_{2}\right)\right. \\
\left.+\tilde{H}_{+}(t) \tilde{H}_{-}\left(t_{1}\right) \exp \left(-i \int_{t_{1}}^{t} V\left(t_{2}\right) d t_{2}\right)\right] S_{z}\left(t_{1}\right) d t_{1}
\end{gathered}
$$

where $\tilde{H}_{ \pm}(t)=\mu_{\nu}\left(B_{x}(t) \pm i B_{y}(t)\right)$ depends on the transversal field components only. The collision frequency $\nu_{\perp}(t)$ is approximately given by the weak elastic collision rate $\Gamma_{W}^{e l}$. In the absence of inelastic collisions the relaxation of the transversal spin components is completely determined by Eq. (34). If $V$ and $\nu_{\perp}$ are slowly varying, one may transform Eq. (34) to a differential equation of third order, after which one may perform the averaging over the random magnetic field by assuming isotropy and using the spectral density representation for the magnetic field correlators. One finds 25] that the probability for helicity change is given by

$$
\begin{aligned}
P_{\nu_{L} \rightarrow \nu_{R}}(t) & =\frac{2 \tilde{H}_{\perp}^{2}}{\omega^{2}}\left[1-\exp \left(-\nu_{\perp} t\right)\left(\cos \omega t+\frac{3 \nu_{\perp}}{\omega} \sin \omega t\right)\right] \\
& +\frac{1}{2}\left[1-\exp \left(-\frac{8 \tilde{H}_{\perp}^{2} \nu_{\perp} t}{\omega^{2}}\right)\right]
\end{aligned}
$$

Note that in the presence of elastic collisions the spin-flip probability increases and tends towards the asymptotic value $1 / 2$ as $t \rightarrow \infty$. In the present section we assumed that all the collisions take place within a homogenous magnetic domain. Hence, when 
evaluating the production rate of right-helicity neutrinos, one should calculate the probability when $t \simeq L_{0}$. During this time the neutrino has been subject to a large number of independent collisions, each of which have served to adjust the spin-content of this state. The scale of the magnetic field felt by the neutrino at each collision is given by the free path length $L_{W}$. Thus we find

$$
\Gamma_{L \rightarrow R}=\frac{4 \tilde{H}_{\perp}^{2} \nu_{\perp} L_{0} \Gamma_{W}^{\text {tot }}}{V^{2}}
$$

Assuming $\nu_{\perp} \approx \Gamma_{W}^{\mathrm{el}}$ it is then straightforward to deduce a constraint on the product $\mu_{\nu} B:$

$$
\mu_{\nu} B\left(T_{\mathrm{QCD}}, L_{W}\right) \lesssim 3.5 \times 10^{2}\left(\frac{L_{W}}{L_{0}}\right)^{1 / 2} .
$$

Note that by taking into account elastic collisions one obtains a limit which is more stringent than in the collisionless case. This is due to the fact that spin rotation turns the longitudinal part of the spin into transversal, and at each collision the transversal part is, in effect, wiped out. This results in a shrinkage of the spin vector and as $t \rightarrow \infty, P_{\nu_{L} \rightarrow \nu_{R}}(t) \rightarrow 1 / 2$. Qualitatively one can also see this in the following manner. Let us write the RKE in the familiar form

$$
\frac{d \mathbf{S}}{d t}=\mathbf{V} \times \mathbf{S}-\nu_{\perp} \mathbf{S}_{\perp}
$$

where now $\mathbf{V}=V \hat{n}_{z}+2 \mu_{\nu}\left(B_{x} \hat{n}_{x}+B_{y} \hat{n}_{y}\right)$ and the transversal spin is given by $S_{\perp} \sim$ $S\left(V_{\perp} / V_{z}\right)$ with $V_{\perp}=2 \mu_{\nu} B_{\perp}$. It then follows that

$$
\frac{d S^{2}}{d t^{2}}=-2 \nu_{\perp} S_{\perp}^{2}
$$

yielding a shrinkage rate $S^{-2} d S^{2} / d t=-\nu_{\perp} \times 8\left\langle\tilde{H}_{\perp}^{2}\right\rangle / V^{2}$, in agreement with Eq. (35).

The usefulness of the limit Eq. (37) in restricting the neutrino magnetic moment depends of course on the magnitude of the primordial magnetic field. Adopting the maximum value allowed by nucleosynthesis, given in Eq. (28), and taking $L_{0} \approx l_{H}$ we see that the tightest possible constraint which in principle can be obtained in this manner is $\mu_{\nu} \lesssim 3 \times 10^{-19} \mu_{B}$. Cosmological [26] and astrophysical [27] constraints on $\mu_{\nu}$, based on the direct production of wrong-helicity neutrinos in photon mediated collisions are typically less severe by several orders of magnitude. Thus the presence of a primordial magnetic field is a potential bonus for neutrino physics.

The derivation of Eq. (37) assumes that $L_{0} \gg \Gamma_{W}^{-1}$. This means that a scattered neutrino meets always a transversal part of a randomly orientated magnetic field. It seems not very likely, however, that the coherence length of the magnetic field at $T=T_{\mathrm{QCD}}$ could be as large as the horizon size, especially if the origin of the field is at earlier times when the size of the horizon was much smaller. Thus, in the next section we consider the corresponding limit in the case of a small scale magnetic field. 


\section{Neutrino propagation in medium with small- scale random magnetic field}

An important technical point in the derivation of Eqs. (32) and (37) is the procedure by which one averages the spin equation of motion over the random magnetic field distribution. For the limits (32) and (37) the exact differential equation derived from Eq. (34) was averaged after it was first transformed to a more suitable form. Such a procedure is always valid for a regular magnetic field, but in the case of random fields we should be more careful. In fact, as we shall now show, in the case of small domains with $L_{0} \ll l_{\text {osc }}$ there appears aperiodic neutrino spin motion.

Let us rewrite Eq. (34) as

$$
\begin{gathered}
\frac{d S_{z}(t)}{d t}=-4 \int_{0}^{t} \exp \left[-\int_{t_{1}}^{t} \nu_{\perp}\left(t_{2}\right) d t_{2}\right] \times\left[\operatorname{Re}\left(\tilde{H}_{-}(t) \tilde{H}_{+}\left(t_{1}\right)\right) \cos \left(\int_{t_{1}}^{t} V\left(t_{2}\right) d t_{2}\right)\right. \\
\left.+\operatorname{Im}\left(\tilde{H}_{+}(t) \tilde{H}_{-}\left(t_{1}\right)\right) \sin \left(\int_{t_{1}}^{t} V\left(t_{2}\right) d t_{2}\right)\right] S_{z}\left(t_{1}\right) d t_{1}
\end{gathered}
$$

Assuming that the collision frequency $\nu_{\perp}$ depends only weakly on the magnetic field, and taking into account that in the leading approximation the potential $V(t)$ as given in Eq. (1) depends on $B_{z}(t)$ only while $\tilde{H}_{ \pm}(t)$ are proportional to the transversal components, we can average these factors in integrand independently because of the isotropy of the system. For neutrinos crossing many small-scale domains with $t \gg L_{0}$, the size $L_{0}$ corresponds to a narrow resonance for uncorrelated random fields, as we now show.

To this end, consider the kernel

$$
K\left(t-t_{1}\right)=\left\langle\tilde{H}_{+}(t) \tilde{H}_{-}\left(t_{1}\right)\right\rangle /\left\langle\tilde{H}_{\perp}^{2}\right\rangle
$$

with

$$
\left\langle\tilde{H}_{\perp}^{2}\right\rangle=(2 / 3) \mu_{\nu}^{2} B^{2} .
$$

If the fields are uncorrelated with $\left\langle B(t) B\left(t_{1}\right)\right\rangle=B^{2} L_{0} \delta\left(t-t_{1}\right)$ one finds

$$
\frac{K(t)}{L_{0}} \sim \lim _{L_{0} \rightarrow 0} \frac{L_{0}}{t^{2}+L_{0}^{2}}=\frac{\pi}{2} \delta(t) .
$$

For such uncorrelated fields the averaging over of the transversal components then results in $\delta\left(t-t_{1}\right)$ under the integral in Eq. (34) which wipes out the exponent in the

\footnotetext{
${ }^{7}$ Here we consider one-dimensional correlators, but the same result could be obtained by using the full 3-d correlator given in Eq. (6). There the factor $\lambda$ is related to the horizon scale because $\left\langle\mathbf{B}^{2}\right\rangle$ involves integration over all space.
} 
integrand and leads (for the initial condition $S_{z}(0)=-1$ ) to a new damping solution

$$
\begin{aligned}
S_{z}(t) & =-\exp (-\Gamma t) \\
P_{\nu_{L} \rightarrow \nu_{R}}(t) & =\frac{(1-\exp (-\Gamma t))}{2} \approx \Gamma t / 2,
\end{aligned}
$$

where the damping parameter $\Gamma$ is given by

$$
\Gamma=\frac{8}{3} \mu_{\nu}^{2} B^{2} L_{0}
$$

One should bear in mind that the averaged field $B$ depends on the horizon scale $L=l_{H}$ and the domain size $L_{0}$ for uncorrelated fields (see Eq. (6)).

As in the previous section, the spin-flip probability grows with time. Now we assumed that $L_{W} \gg L_{0}$ so that we should calculate the probability at largest possible time. Setting $t=H^{-1}$ we find

$$
\Gamma_{L \rightarrow R}=\frac{4}{3} \mu_{\nu}^{2} B^{2} L_{0} H^{-1} \Gamma_{W}^{\mathrm{tot}}
$$

Note that $\Gamma_{L \rightarrow R} \sim T^{3}$, so that again we should require $\Gamma_{L \rightarrow R}<H$ at $T=T_{\mathrm{QCD}}$. This results in the bound

$$
\mu_{\nu} B\left(T_{\mathrm{QCD}}, l_{H}\right) \lesssim 6.7 \times 10^{-3} \mu_{B} \mathrm{G}\left(\frac{L_{W}}{L_{0}}\right)^{1 / 2} .
$$

Note that this bound does not agree with Eq. (37) in the limit $L_{0} \rightarrow L_{W}$. This is due to the qualitatively different averaging procedures at large and small $L_{0}$. When $L_{0} \approx L_{W}$, neither method is reliable.

Substituting Eq. (5) to Eq. (48) we can rewrite the BBN constraint on the Dirac neutrino magnetic moment as

$$
\mu_{\nu} \lesssim \frac{1.7 \times 10^{-21} \mu_{B}}{\left(L_{0}^{\min }\right)^{p+1 / 2} \Gamma_{W}^{1 / 2} H^{p}}
$$

where the minimum domain scale $L_{0}^{\min }\left(T_{Q C D}\right)$, the left neutrino total collision rate $\Gamma_{W}\left(T_{\mathrm{QCD}}\right)$ as given by Eq. (31), and the Hubble parameter $H\left(T_{\mathrm{QCD}}\right)$ are the functions of the temperature $T_{\mathrm{QCD}}=200 \mathrm{MeV} \times T_{200}$.

As we have discussed in section 2 there are two scenarios for the choice of the minimum scale $L_{0}^{\min }(T)$. Let us first assume that the relic field is the seed for the galactic magnetic field, so that the domains with a size $L_{0} \geq L_{0}^{\min }(T)=10^{3} \mathrm{~cm}(\mathrm{MeV} / T)$ survive after the recombination time. In the second scenariof, with the use of the

\footnotetext{
${ }^{8}$ The first scenario is not necessary since there are other possibilities for the seed field creation in the MHD-dynamo theory of the galactic magnetic fields.
} 
relativistic plasma dissipation length (16), the domains with sizes $L_{0} \geq L_{0}^{m i n^{\prime}}=$ $10^{-2} \mathrm{~cm}(\mathrm{MeV} / T)$ survive at any relativistic temperature $T \gtrsim 2 \mathrm{MeV}$, but dissipate even before the BBN temperature $T \sim 0.1 \mathrm{MeV}$. This last fact does not matter for the neutrino spin-flip that populates wrong helicity states mainly around $T \sim T_{\mathrm{QCD}}$. Thus, we find from Eq. (49) two upper limits for the Dirac neutrino magnetic moment:

$$
\mu_{\nu} \lesssim \frac{10^{-22+6 p} \mu_{B}}{(5.4)^{p} T_{200}^{p+2}},
$$

for the first case with the relic seeding of galactic magnetic fields, and

$$
\mu_{\nu} \lesssim \frac{3.2 \times 10^{-20+11 p} \mu_{B}}{(5.4)^{p} T_{200}^{p+2}}
$$

for the second scenario with dissipation of the random fields before the BBN time.

As an example, for the index $p=1 / 2$ we obtain from Eqs. (50) and (51) very restrictive constraints on the Dirac neutrino magnetic moments, $\mu_{\nu} \lesssim 4.3 \times 10^{-20} \mu_{B} / T_{200}^{5 / 2}$, or $\mu_{\nu} \lesssim 4.4 \times 10^{-15} \mu_{B} / T_{200}^{5 / 2}$, respectively. These numbers are deduced by requiring that there should not occur full equilibration of one right-handed neutrino species. If we require that $\Delta N_{\nu} \lesssim 0.1$, we should multiply the upper limits above by a factor $\left(\Delta N_{\nu}\right)^{-1 / 2} \sim 3$. However, we wish to emphasize that the very stringent constraints on the Dirac magnetic moment above are very sensitive to the model of the primordial magnetic field. We should also point out that the both scenarios are based on the common assumption that the magnetic coherence length is much larger than the interparticle distance, $L_{0} \gg T^{-1}$, an assumption which is natural considering the macroscopic nature of the "glueing" of the magnetic field force lines on hot plasma.

The remaining issue is the validity of the RKE Eq. (34) when also inelastic collisions are taken into account. This work is now in progress.

\section{$5 \quad$ Discussion and conclusions}

We have found out that the general constraints on the primordial magnetic fields, as implied by nucleosynthesis, do not exclude the possibility of very tight limits on the Dirac neutrino magnetic moments in the presence of magnetic fields. These can be derived from the requirement that the right-handed components should not be in thermal equilibrium at time of nucleosynthesis. We found, however, that nucleosynthesis and the dynamo origin of the observed galactic magnetic fields are not compatible with a magnetic field model consisting of uncorrelated cells with a scaling index $p=3 / 2$, and only marginally compatible if $p=1$. If the field reversals observed in the Milky Way 
are due to the dynamo, then only the scaling law $p \lesssim 1 / 2$ is appropriate (this naturally includes the constant background $p=0$ as in the case of the Savvidy vacuum [6]).

The particle physics aspect of the magnetic moment constraints is straightforward: one only needs to know the neutrino collision rates just above the QCD phase transition. Neutrino spin evolution is then determined by a general RKE. Here we included only the effects due to the elastic scattering. Generally speaking, we should also account for the dependence of the weak rates on the strong magnetic field. For the spin collision integrals this problem is now in progress, together with the generalization of the RKE (34) to the case of inelastic collisions. It is not obvious how ineleastic collisions affect the evolution of the neutrino spin.

The actual limits on the neutrino magnetic moments depend on two unknowns: the strength of the field at $T \approx T_{\mathrm{QCD}}$, and the size of the homogenous magnetic field domain $L_{0}$. The magnitude of the field depends on the mechanism by which it was first produced, at the electroweak phase transition or earlier, and on its scaling when averaged over several domains, if $L_{0} \ll \Gamma_{W}^{-1}$. In a given model of the primordial magnetic field both these can be estimated, but here we adopted a phenomenological view and simply assumed $B \sim\left(L_{0} / L\right)^{p}$. The magnitude of $L_{0}$ is a more complicated issue as it involves magnetohydrodynamics in the hot plasma of the early universe. It seems obvious, though, that $L_{0} \gg 1 / T$ as the build-up of the magnetized plasma requires a large number of particles, but that $L_{0}$ is (much) less that the horizon size. One possibility would be that the collision frequency of charged particles (i.e conductivity) plays a decisive role in forming homogenous regions. This remains an open problem. In the present work we studied the cases with $L_{0} \ll \Gamma_{W}^{-1}$ and $L_{0} \gg \Gamma_{W}^{-1}$, which lead to qualitatively different evolution equations. We also gave some examples of the possible order of magnitude of the upper limits on $\mu_{\nu}$ which could turn out to be as restrictive as $10^{-20} \mu_{B}$. The limit also depends on whether the primordial field provides the seed for galactic dynamo, and thus in the absence of a more detailed knowledge of the dynamics of the primordial magnetic fields no definite statement about the actual constraint on neutrino magnetic moments can be made. We may however conclude that potentially the primordial magnetic field constraint on Dirac neutrinos could be very important.

Acknowledgements A. Rez would like to thank The Education and Research Center "Cosmion" for initiative grant. V. Semikoz acknowledges the High Energy Physics group of the Research Institute for Theoretical Physics at the Helsinki University for hospitality during the preparation of this work. We thank J. Maalampi for fruitful discussions. 


\section{References}

[1] Ya.B. Zeldovich, A.A. Ruzmaikin and D.D. Sokoloff, Magnetic Fields in Astrophysics (McGraw-Hill, New York, 1980); E.N. Parker, Cosmological Magnetic Fields (Oxford Univ. Press, Oxford, 1979); A.A. Ruzmaikin, A.A. Shukurov and D.D. Sokoloff, Magnetic Fields of Galaxies (Kluwer, Dordrecht, 1988).

[2] C.J. Hogan, Phys. Rev. Lett. 51 (1983) 1488; M.S. Turner and L.W. Widrow, Phys. Rev. D37 (1988) 2743; B. Ratra, Astrophys. J. Lett. 391 (1992) L1.

[3] T. Vachaspati, Phys. Lett. B265 (1991) 258; P. Olesen, Phys. Lett B281 (1992) 300.

[4] K. Enqvist and P. Olesen, Phys. Lett. B319 (1993) 178.

[5] See e.g. A. Brandenburg et al., Astron. Astrophys. 271 (1993) 36, and references therein.

[6] K. Enqvist and P. Olesen, Phys. Lett. B329 (1994) 195.

[7] B. Cheng, D.N. Schramm and J.W. Truran, Phys. Rev. D49 (1994) 5006.

[8] K. Enqvist, P. Olesen and V. Semikoz, Phys. Rev. Lett. 69 (1992) 2157.

[9] K. Olive and G. Steigman, preprint UMN-TH-1230/94.

[10] K. Enqvist and V. Semikoz, Phys. Lett. B312 (1993) 310.

[11] V. Semikoz, Phys. Rev. D48 (1993) 5264.

[12] V. Semikoz and J.W.F.Valle, Valencia Preprint FTUV/94-05, to appear in Nucl. Phys. B.

[13] D. Nötzold and G. Raffelt, Nucl. Phys. B307 (1988) 924; K. Enqvist, K. Kainulainen and J. Maalampi, Nucl. Phys. B349 (1991) 754

[14] A.I. Akhiezer et. al, Plasma Electrodynamics, vol 2, Non-linear theory and Fluctuations, Pergamon Press (1975) p. 117.

[15] V.D. Shafranov, Electromagnetic waves in a plasma in Reviews of plasma Physics, ed. by Acad. M.A. Leontovich, vol 3, Consultants Bureau, New York (1967) p. 140.

[16] S. Langer, Phys. Rev. D23 (1981) 328. 
[17] R. Gonzales Felipe, A. Perez Martinez and H. Perez-Rojas, Mod. Phys. Lett. 4 (1990) 1103.

[18] A.I. Akhiezer et al, Plasma Electrodynamics, vol 1, Linear Theory Pergamon Press (1975) p. 25.

[19] K. Enqvist, V. Semikoz, A. Shukurov and D. Sokoloff, Phys. Rev. D48 (1993) 4557.

[20] M. Simard-Normandin and P.P. Kronberg, Nature 229 (1979) 115; Astrophys. J. $242(1980) 74$.

[21] A. Poezd, A. Shukurov and D. Sokoloff, Mon. Not. R. Astron. Soc. 264 (1993) 285.

[22] M. Bander and H. Rubinstein, Phys. Lett. B311 (1993) 187.

[23] T.Walker, G.Steigman, D.N.Schramm, K.Olive, and H.Kang, Astrophys. J. 376 (1991) 51.

[24] K. Enqvist and H. Uibo, Phys. Lett. B301 (1993) 376.

[25] V. Semikoz, Valencia Preprint IFIC/93-32 (1993).

[26] M. Fukugita and S. Yazaki, Phys. Rev. D36 (1987) 3817.

[27] G. Raffelt, Phys. Rep. 198 (1990) 1. 